ISSN 2075-4701

www.mdpi.com/journal/metals/

\title{
Editorial
}

\section{Metallic Glasses}

\section{Kang Cheung Chan ${ }^{1, *}$ and Jordi Sort ${ }^{2, *}$}

1 Advanced Manufacturing Technology Research Centre, Department of Industrial and Systems Engineering, The Hong Kong Polytechnic University, Hung Hom, Kowloon, HK

2 Institució Catalana de Recerca i Estudis Avançats (ICREA) and Departament de Física, Universitat Autònoma de Barcelona, E-08193 Bellaterra, Spain

* Authors to whom correspondence should be addressed;

E-Mails: kc.chan@polyu.edu.hk (K.C.C.); jordi.sort@uab.cat (J.S.);

Tel.: +852-2766-4981 (K.C.C.); +34-935812085 (J.S.); Fax: +34-935812155 (J.S).

Received: 15 December 2015 / Accepted: 15 December 2015 / Published: 17 December 2015

Metallic glasses are a fascinating class of metallic materials that do not display long-range atomic order. Due to their amorphous character and the concomitant lack of dislocations, these materials exhibit mechanical properties that are quite different from those of other solid materials [1,2]. For example, they can be twice as strong as steels, show more elasticity and fracture toughness than ceramics and be less brittle than conventional oxide glasses. In addition to their unique mechanical properties, metallic glasses have also demonstrated interesting physical and chemical properties. For example, some metallic glasses have been found to exhibit superior soft magnetic properties [3], good magnetocaloric effects [4] and outstanding catalytic performance [5], thus having potential for a widespread range of technological applications [6].

Metallic glasses become relatively malleable when heated in the supercooled liquid region, allowing moulding and shaping with microscale precision by means of thermoplastic processing [7]. This has facilitated the development of diverse products based on these alloys, including sporting goods, medical and electronic devices and advanced defence and aerospace applications. However, in spite of their large elasticity, metallic glasses exhibit poor room-temperature macroscopic plasticity compared to polycrystalline metals [8]. This low plastic deformation, particularly evidenced when testing metallic glasses under tension, is related to the formation and rapid propagation of shear bands [2]. Therefore, novel routes to enhance plasticity of metallic glasses include procedures to hinder shear band propagation. This can be achieved, for example, by designing composite materials consisting of particles which act as second-phase reinforcements embedded in the amorphous matrix [9,10]. Other approaches towards toughening of metallic glasses have also been developed, such as the preparation of the so-called dual-phase amorphous metals [11], some specific surface treatments (e.g., laser or shot pinning) [12], or making their overall structure porous (i.e., metallic glass foams) [13]. 
The aim of this Special Issue is to address, from both experimental and theoretical points of view, some of the challenges to improve the glass forming ability of metallic glasses, to optimize their overall mechanical performance, and to enhance their physico-chemical properties. The intricate dynamic and geometrical features of the icosahedral atomic clusters arrangements in liquid and amorphous states in $\mathrm{Zr}-\mathrm{Cu}$ alloys are investigated by $\mathrm{M}$. Shimono and $\mathrm{H}$. Onodera using molecular dynamics simulations [14]. In spite of the lack of long-range order, the simulations reveal that such icosahedral clusters induce medium range order in these alloys. The atomic structure frozen-in during the ultra-rapid cooling processes needed to generate metallic glasses is usually not stable at room temperature. V. A. Khonik reviews how the presence of interstitialcy defects and the change in their concentration can induce structural relaxation when metallic glasses are heat treated to temperatures below or around the glass transition [15]. Such structural relaxation is important since it can induce changes in many physical properties of the glasses, particularly in the mechanical behavior (elasticity, anelasticity, viscoelasticity, etc. [16]), but also in the electrical, corrosion and even magnetic performances. Of course, atomic rearrangements of the glassy structure take place also during mechanical treatments. This can result in mechanically-induced structural relaxation, as reviewed by C. Liu et al. [17]. Dynamic mechanical measurements as a function of temperature reveal that structural relaxation is a complex phenomenon that depends on the frequency of the treatments. In fact, one can distinguish between primary and secondary relaxation effects, both of which are relevant for a full understanding of the complex and unique properties of amorphous metallic alloys.

As it might be anticipated, the properties of metallic glasses not only depend on their structure (i.e., medium-range order, presence of flow defects, etc.) but also on the actual composition of the glasses. T. Bitoh and D. Watanabe show, for example, that incorporation of $\mathrm{Y}$ in the amorphous structure of $\mathrm{Fe}-\mathrm{Co}-\mathrm{B}-\mathrm{Si}-\mathrm{Nb}$ bulk metallic glasses induces changes in their glass forming ability and the magnetic properties of these alloys [18]. The properties of newly developed Ni-Cu-W-B metallic glasses are reported by A. Hitit et al. [19]. The current theories describing the links between toughness and material parameters, including elastic constants, alloy chemistry and ordering degree in the glass are reviewed by S. V. Madge [20]. As aforementioned, metallic glasses are relatively brittle when tested in their fully amorphous as-prepared state. Strategies to increase plastic deformation based partial crystallization or formation of metallic glass composites are reported by D. Wu et al. [21] and Ö. Balc1 et al. [22]. Formation of metallic glass porous frameworks is described by B. Sarac et al. [23]. Finally, the interplay between corrosion effects and mechanical properties of Zr-based metallic glasses are surveyed by P.F. Gostin et al. [24].

Although the plasticity of metallic glasses in bulk form is rather limited due to the rapid propagation of single shear bands [1,2], an improved mechanical performance has been reported in miniaturized metallic glasses $[25,26]$. For this reason, there is a growing interest in new approaches towards the synthesis of metallic glass microwires [27] and nanowires [26], in view of their potential applications in micro/nano-electro-mechanical systems (MEMS/NEMS). From the aforementioned aspects and the ongoing works on the topic, it is likely that the interest in metallic glasses will continue to increase in the near future with the development of new compositions and novel applications, particularly in devices with micrometer and submicrometer sizes, where the full potential of these glassy materials is yet to come. 


\section{Acknowledgments}

This work was supported by the "Ministerio de Economía y Competitividad" through project MAT2014-57960-C3-1-R (co-financed by the Fondo Europeo de Desarrollo Regional (FEDER)). Partial financial support from the 2014-SGR-1015 project from D.G.U. Catalunya is also acknowledged.

\section{Conflicts of Interest}

The authors declare no conflict of interest.

\section{References}

1. Schuh, C.A.; Hufnagel, T.C.; Ramamurty, U. Mechanical behavior of amorphous alloys. Acta Mater. 2007, 55, 4067-4109.

2. Greer, A.L.; Cheng, Y.Q.; Ma, E. Shear bands in metallic glasses. Mater. Sci. Eng. R 2013, 74, 71-132.

3. Suryanarayana, C.; Inoue A. Iron-based bulk metallic glasses. Int. Mater. Rev. 2013, 58, 131-166.

4. Xia, L.; Chan, K.C.; Tang, M.B. Enhanced glass forming ability and refrigerant capacity of a $\mathrm{Gd}_{55} \mathrm{Ni}_{22} \mathrm{Mn}_{3} \mathrm{Al}_{20}$ bulk metallic glass. J. Alloys Compd. 2011, 509, 6640-6643.

5. Zhao, M.; Abe, K.; Yamaura, S.-I.; Yamamoto, Y.; Asao, N. Fabrication of Pd-Ni-P metallic glass nanoparticles and their application as highly durable catalysts in methanol electro-oxidation. Chem. Mater. 2014, 26, 1056-1061.

6. Inoue, A.; Wang, X.M.; Zhang, W. Developments and applications of bulk metallic glasses. Rev. Adv. Mater. Sci. 2008, 18, 1-9.

7. Kumar, G.; Tang, H.X.; Schroers, J. Nanomoulding with amorphous metals. Nature 2009, 457, 868-872.

8. Fornell, J.; Concustell, A.; Suriñach, S.; Li, W.H., Cuadrado, N.; Gebert, A.; Baró, M.D.; Sort, J. Yielding and intrinsic plasticity of $\mathrm{Ti}-\mathrm{Zr}-\mathrm{Ni}-\mathrm{Cu}-\mathrm{Be}$ bulk metallic glass. Int. J. Plast. 2008, 25, 1540-1559.

9. Hofmann, D.C.; Suh, J.-Y.; Wiest, A.; Duan, G.; Lind, M.-L.; Demetriou, M.D.; Johnson, W.L. Designing metallic glass matrix composites with high toughness and tensile ductility. Nature 2008, 451, 1085-1089.

10. Wu, F.F.; Chan, K.C.; Jiang, S.S.; Chen, S.H.; Wang, G. Bulk metallic glass composite with good tensile ductility, high strength and large elastic strain limit. Sci. Rep. 2014, 4, 5302.

11. Concustell, A.; Mattern, N.; Wendrock, H.; Kuehn, U.; Gebert, A.; Eckert, J.; Greer, A.L.; Sort, J.; Baró, M.D. Mechanical properties of a two-phase amorphous $\mathrm{Ni}-\mathrm{Nb}-\mathrm{Y}$ alloy studied by nanoindentation. Scr. Mater. 2007, 56, 85-88.

12. González, S.; Fornell, J.; Pellicer, E.; Suriñach, S.; Baró, M.D.; Greer, A.L.; Belzunce, F.J.; Sort, J. Influence of the shot-peening intensity on the structure and near-surface mechanical properties of $\mathrm{Ti}_{40} \mathrm{Zr}_{10} \mathrm{Cu}_{38} \mathrm{Pd}_{12}$ bulk metallic glass. Appl. Phys. Lett. 2013, 103, 211907.

13. Brothers, A.H.; Dunand, D.C. Ductile bulk metallic glass foams. Adv. Mater. 2005, 17, 484-486.

14. Shimono, M.; Onodera, H. Dynamics and geometry of icosahedral order in liquid and glassy phases of metallic glasses. Metals 2015, 5, 1163-1187. 
15. Khonik, V.A. Understanding of the structural relaxation of metallic glasses within the framework of the interstitialcy theory. Metals 2015, 5, 504-529.

16. Van Steenberge, N.; Sort, J.; Concustell, A.; Das, J.; Scudino, S.; Suriñach, S.; Eckert, J.; Baró, M.D. Dynamic softening and indentation size effect in a Zr-based bulk glass-forming alloy. Scr. Mater. 2007, 56, 605-608.

17. Liu, C.; Pineda, E.; Crespo, D. Mechanical relaxation of metallic glasses: An overview of experimental data and theoretical models. Metals 2015, 5, 1073-1111.

18. Bitoh, T.; Watanabe, D. Effect of yttrium addition on glass-forming ability and magnetic properties of Fe-Co-B-Si-Nb bulk metallic glass. Metals 2015, 5, 1127-1135.

19. Hitit, A.; Şahin, H.; Öztürk, P.; Aşgın, A.M. A new Ni-based metallic glass with high thermal stability and hardness. Metals 2015, 5, 162-171.

20. Madge, S.V. Toughness of bulk metallic glasses. Metals 2015, 5, 1279-1305.

21. Wu, D.; Song, K.; Cao, C.; Li, R.; Wang, G.; Wu, Y.; Wan, F.; Ding, F.; Shi, Y.; Bai, X.; et al. Deformation-induced martensitic transformation in $\mathrm{Cu}-\mathrm{Zr}-\mathrm{Zn}$ bulk metallic glass composites. Metals 2015, 5, 2134-2147.

22. Balc1, Ö.; Prashanth, K.G.; Scudino, S.; Ağaoğulları, D.; Duman, İ.; Öveçoğlu, M.L.; Uhlenwinkel, V.; Eckert, J. Effect of milling time and the consolidation process on the properties of Al matrix composites reinforced with Fe-based glassy particles. Metals 2015, 5, 669-685.

23. Sarac, B.; Sopu, D.; Park, E.; Hufenbach, J.K.; Oswald, S.; Stoica, M.; Eckert, J. Mechanical and structural investigation of porous bulk metallic glasses. Metals 2015, 5, 920-933.

24. Gostin, P.F.; Eigel, D.; Grell, D.; Uhlemann, M.; Kerscher, E.; Eckert, J.; Gebert , A. Stress-corrosion interactions in Zr-based bulk metallic glasses. Metals 2015, 5, 1262-1278.

25. Ashby, M.F.; Greer, A.L. Metallic glasses as structural materials. Scr. Mater. 2006, 56, 321-326.

26. Zeeshan, M.A.; Esqué-de los Ojos, D.; Castro-Hartmann, P.; Guerrero, M.; Nogués, J.; Suriñach, S.; Baró, M.D.; Nelson, B.J.; Pané, S.; Pellicer E.; et al. Electrochemically synthesized amorphous and crystalline nanowires: Dissimilar nanomechanical behavior in comparison with homologous flat films. Nanoscale 2016, doi:10.1039/C5NR04398K.

27. Olofinjana, A.; Voo, N.Y. On the stability of the melt jet stream during casting of metallic glass wires. Metals 2015, 5, 1029-1044.

(C) 2015 by the authors; licensee MDPI, Basel, Switzerland. This article is an open access article distributed under the terms and conditions of the Creative Commons Attribution license (http://creativecommons.org/licenses/by/4.0/). 